\title{
Rationales Operationsmanagement verbessert Ergebnisse bei Tibiakopffrakturen
}

(ur n jahrzehntelangen Anstrengungen zur Verfeinerung anatomischer und physiologischer Kenntnisse hat sich eine starke operative Bereitschaft zu primären und sekundären Rekonstruktionen bestimmter Kniebandverletzungen bei Patienten und Ärzten entwickelt, deren allgemeiner Nutzen noch nachzuweisen ist. - Nach enthusiastischer Verbreitung systematisierter Osteosynthesetechniken ist hingegen die operative Behandlung der Tibiakopffrakturen in Mißkredit geraten, da sie von einer hohen Anzahl von Mißerfolgen begleitet war (vorwiegend deshalb, weil nicht genügend beachtet wurde, daß das Knochengewebe Blutgefäße und Weichteile zum Überleben braucht). Die epidemische Belegung nahezu jeder Osteosynthese mit dem Beiwort „biologisch“ ist Eingeständnis dieses falschen Weges. Zusammen mit Korrekturen der Operationstaktik, der Operationstechnik und Verminderung des Implantatvolumens bei gleichzeitiger Übungsstabilität hat sich eine Renaissance der Osteosynthese der Tibiakopffrakturen eingestellt, die in der Komplikationsstatistik an die be- staunten Ergebnisse der konservativen Behandlung von Jahna heranreicht. Verbesserte Bildgebung, direkte arthroskopische Beobachtung und nicht zuletzt gute Kenntnisse der besonderen Ausformung des Tibiakopfes sind zusätzliche wertvolle Hilfen.

Minimal-invasives Vorgehen ist bei Tibiakopffrakturen kein Dogma, sondern besonders unter Verwendung arthroskopischer Techniken eine kleine Erweiterung operationstechnischer Möglichkeiten, die im Ergebnis keine Verbesserung gegenüber einer technisch und taktisch einwandfreien "offenen " $\mathrm{Ge}$ lenkosteosynthese bietet, wohl aber die Gefahren der Überstrapazierung mit optischen Fehlern, zeitlicher Ausdehnung und anatomischen Irrtümern in sich birgt - dies auch bei in der Arthroskopie erfahrenen Operateuren. Kein Chirurg soll sich durch publizistischen Druck verleiten lassen, gute Erfolge auf schwierigere Weise erzielen zu wollen. Bei den minimal-invasiven Verfahren ist die integrierte Anwendung des Bildverstärkers obligat und für eine genaue Reposition wichtiger als das Arthroskop.

Eine ganz andere Problematik entsteht bei der Behandlung von Tibiakopffrakturen bei älteren Patienten. Probleme der Knochenfestigkeit, der Implantatverankerung, der Belastung während der Mobilisation und der Re- vaskularisierung stehen hier im Vordergrund. Die Grenzbereiche der konservativen Behandlung müssen hier bewußt und individuell weiter gesteckt sein. Stabile Impaktierungen mit einer Fehlstellung sind hier besser als unsichere, gut ausgerichtete Osteosynthesen.

Ein bewußtes, rationales Komplikationsmanagement schafft den vorausschauenden Blick für mögliche Komplikationen und hilft von vornherein, diese zu vermeiden. Fehlstellungen nach Tibiakopffrakturen sind ein ungelöstes Problem (wie hoch sind tolerable Gelenkstufen? Wie früh soll man eine Osteosyntehse korrigieren?). Die Frage der Defektfüllung ist bewußt einseitig beantwortet - die Diskussion um Knochenersatzmaterialien wird an anderer Stelle grundsätzlicher geführt.
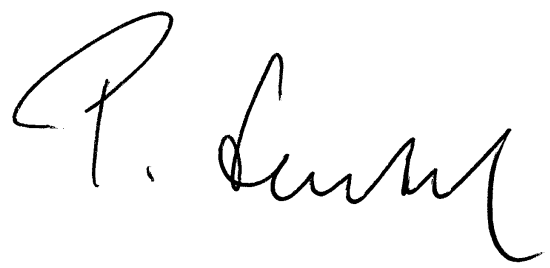